\title{
Can We Obtain a Noninvasive and Continuous Estimation of Cardiac Output? Comparison Between Three Noninvasive Methods
}

\author{
Zainab Raissuni, ${ }^{1 *}$ MD, Florian Zores,${ }^{1 *} \mathrm{MD}$, Odile Henriet,${ }^{1 *} \mathrm{RN}$, \\ Stephanie Dallest, ${ }^{1 *} \mathrm{RN}$, and Gerald Roul, ${ }^{1 *} \mathrm{MD}$
}

\begin{abstract}
SUMMARY
Cardiac output $(\mathrm{CO})$ is often desirable for assessing the hemodynamic condition of a patient, especially in critically ill cardiac patients. Various noninvasive methods are available for this purpose. Inert gas rebreathing (IGR) and 2D-Doppler echocardiography methods have been validated. Based on the relationship between pulse wave transit time and stroke volume, the VISMO ${ }^{\circledR}$ provides an estimated continuous cardiac output (esCCO) measurement using only an electrocardiogram, pulse oximeter wave, and cuff arterial blood pressure. Doppler echocardiography is being currently used in every day practice in this setting and IGR is a validated method, thus we wanted to assess the agreement between these 3 methods for noninvasive CO calculation and reproducibility of esCCO. Patients followed in our cardiology department received on the same day a CO analysis by esCCO, Doppler echocardiography and IGR. Thirty-four patients were included (16 women, mean age $65 \pm 15$ years). Bland and Altman plots showed a good agreement between IGR and 2D-Doppler echocardiography (bias $=0.31 \mathrm{~L} /$ minute). Though there was also an agreement between esCCO and the other 2, the bias was rather large: $1.18 \mathrm{~L} /$ minute with IGR and $1.51 \mathrm{~L} / \mathrm{min}$ with 2D-Doppler echo. The intraclass correlation coefficient was poor whatever the methods. However, esCCO had a satisfactory reproducibility and accuracy compared rather well with the other 2. This method could be suitable for patient screening and monitoring. (Int Heart $\mathrm{J}$ 2013; 54: 395-400)
\end{abstract}

Key words: Pulse wave transit time, Inert gas rebreathing, 2D doppler echocardiography

$\mathrm{T}$

he incidence of heart failure is growing as underscored by various reports. ${ }^{1-2)}$ In the outpatient setting, those with this condition are evaluated using imaging (ie, Doppler echocardiography), biomarkers - mainly natriuretic peptide - and analysis of exercise tolerance (walking test or cardiopulmonary exercise testing). These data give access not only to the patient's actual clinical condition but also to their prognosis. ${ }^{3)}$ All these techniques are related to hemodynamic status. Direct assessment of the hemodynamic parameters is less frequently performed nowadays, especially since publication of the ESCAPE study. ${ }^{4)}$ However, hemodynamic followup is still of value during general anesthesia or in the critical care unit. ${ }^{5,6}$ The availability of hemodynamic data is also paramount in cases of acute HF or in HF with high cardiac output. For prognostic purposes, apart from filling pressures, the value of cardiac power was also underlined. ${ }^{7)}$ Thus, having direct access to the hemodynamic parameters might be worth considering if these could be obtained noninvasively, reliably, rapidly, and at the lowest cost possible. The value of intracardiac filling pressure monitoring is well established. Cardiac output (CO) reflects, at least to some extent, filling pressure trends but also patient intravascular volume status (ie, cardiac preload) and the hemodynamic response after fluid challenge (ie, volume responsiveness). Various noninvasive methods have been developed to measure $\mathrm{CO} .{ }^{8)}$ 2D-Doppler echocardiography and inert gas rebreathing (IGR) have been validated as reliable tools for computing CO. Apart from accuracy, the reproducibility of noninvasive methods is important. Several noninvasive techniques including Doppler echocardiography have failed to show sufficient accuracy to guide therapy. Ishihara, et $a l^{9)}$ proposed a new method to assess cardiac output: estimated continuous cardiac output (esCCO) measurement using pulse wave transit time (PWTT). The concept of pulse contour analysis is based on the relation among blood pressure (BP), stroke volume (SV), arterial compliance, and systemic vascular resistance (SVR). The SV or CO can be calculated from the arterial pressure waveform if the arterial compliance and SVR are known. Arterial waveform-based $\mathrm{CO}$ requires arterial puncture. PWTT has good correlation with SV. It consists of a preejection period, pulse wave transit time through the artery, and pulse wave transit time through the peripheral arteries, which compensates for the effect of changes in SVR. Based on the relationship between PWTT and SV, the VISMO ${ }^{\circledR}$ provides an estimated continuous $\mathrm{CO}$ (esCCO) measurement using an

From the ${ }^{1}$ Pôle d'Activité Médico-Chirurgicale Cardiovasculaire, Unité de prise en charge de I'insuffisance cardiaque et des cardiomyopathies Nouvel Hôpital Civil, Strasbourg, France

*These authors contributed equally to this work.

Address for correspondence: Zainab Raissuni, MD, Pôle d'Activité Médico-Chirurgicale Cardiovasculaire, Unité de prise en charge de I'insuffisance cardiaque et des cardiomyopathies, Nouvel Hôpital Civil, 1, Place de l'Hôpital 67000 Strasbourg, France

Received for publication May 18, 2013.

Revised and accepted June 13, 2013. 
electrocardiogram, pulse oximeter wave, and cuff arterial BP. The esCCO gives access to many hemodynamic parameters including $\mathrm{CO}$. Validation of this new technology compared favourably against the thermodilution method. ${ }^{10)}$ The agreement was considered adequate although the bias was 0.13 and precision $1.15 \mathrm{~L} / \mathrm{min}$. PWTT has the great advantage of simplifying $\mathrm{CO}$ measurement as it is easy to use, rather inexpensive, and requires no consumables. ${ }^{11)}$ To the best of our knowledge, no comparison has been made between esCCO, IGR, and Doppler echocardiography, which is the most widely used method in every day practice in this setting. The purpose of this study was to verify the accuracy and reproducibility of esCCO compared to 2D Doppler echocardiography and IGR.

\section{Methods}

Patients referred to our noninvasive cardiovascular laboratory from January to April 2012 were prospectively considered. The only inclusion criterion was the availability on the same day of the following tests: an assessment of $\mathrm{CO}$ by inert gas rebreathing method, an esCCO analysis, and 2D-echocardiography. These tests together with blood sample analyses are routinely performed in our facility either for HF patients or in patients referred for a thorough cardiovascular investigation before resuming physical activity. There were no exclusion criteria. The study complied with the Helsinki Declaration and informed consent was obtained from the participants prior to the study.

Past medical history and cardiovascular risk factors were recorded. Ongoing drug treatment and functional class were also noted and some vital signs measured: body mass index, pulse oximetry, resting brachial $\mathrm{BP}$, respiratory frequency, and heart rate. All measurements of $\mathrm{CO}$ were performed in a quiet temperature-controlled room to limit variations as much as possible. Between 2D-Doppler echocardiography or esCCO and IGR, a 30-minute rest interval was observed in order to limit fluctuations in the condition of the patient as much as possible.

PWTT-derived CO: This technique provides noninvasive, real-time, continuous calculation of $\mathrm{CO}$ from the pulsatile pressure curve and the R wave. Briefly, patients in the dorsal decubitus position were monitored using a VISMO ${ }^{\circledR}$ (Nihon Koden $\mathrm{GmbH}$, Rosbach, Germany) cardiac monitor equipped with 3 electrodes on the chest wall for cardiac rhythm analysis, a pulse oximetry sensor placed on the middle finger, and a brachial BP cuff. PWTT was computed from pulse oximetry and $\mathrm{R}$ wave from the ECG. Estimated continuous $\mathrm{CO}$ (esCCO) was derived as follows: esCCO $=(\mathrm{K} \times \mathrm{PP}) \times \mathrm{HR}(\mathrm{SV}=$ stroke volume, $\mathrm{K}=$ constant value; $\mathrm{PP}=$ pulse pressure, $\mathrm{HR}=$ heart rate). A better correlation between PWTT and SV was reported so the formula was modified accordingly. Therefore, the new formula was esCCO $=\mathrm{K} \times(\alpha \times \mathrm{PWTT}+\beta) \times \mathrm{HR} ;(\mathrm{K}, \alpha$ and $\beta$ are experimental constants). ${ }^{11)} \mathrm{ECG}$ data, pulse oximetry, systolic, diastolic and mean BP as well as respiratory frequency and esCCO were obtained from the VISMO ${ }^{\circledR}$ cardiac monitor and 5 datasets were averaged for our purpose.

CO measurement by inert gas rebreathing method: The measurement was performed using the Innocor ${ }^{\circledR}$ system (Innovision $\mathrm{GmbH}$, Kaltenkirchen, Germany) as described elsewhere ${ }^{12)}$ : the Innocor ${ }^{\circledR}$ uses rapid photo-acoustic or magneto-acoustic measurement of tidal gas concentrations. The device incorporates an automated rebreathing valve and rebreathing bag, which fills prior to start of the rebreathing maneuver to a calibrated volume of up to $2 \mathrm{~L}$, with a mixture containing approximately $0.5 \% \mathrm{~N}_{2} \mathrm{O}$ (soluble gas) and $0.1 \%$ sulfur hexafluoride (SF6; insoluble gas). Subjects had a nose clip applied, and breathed into the rebreathing system at a rate of approximately 20 breaths/minute with a tidal volume sufficient to empty the bag with each inspiration. This triggered switching of the rebreathing valve to the bag and rebreathing of the contents of the bag, which continued until automatically terminated when tidal variations in insoluble gas concentrations indicated adequate mixing of gas between lung and bag, or after approximately 30 seconds if this was not fully achieved. A pulse oximeter allows the determination of $\mathrm{SpO}_{2}$. Each patient in the sitting position performed 2 measurements of $\mathrm{CO}$ at 5-minute intervals. Incorrect values were excluded and valid data were averaged for our purpose. BP was also measured using an automatic device (Microlife AG, BP A90; Widnau, Switzerland). We used IGR as a standard, as it has already been compared to Dopple echocardiography and arterial pulse analysis. ${ }^{13,14)}$

2D-Doppler echocardiography: 2D-Doppler echocardiography (Vivid 7, General Electric, Milwaukee, WI, USA) was performed in the subsample of diseased patients $(n=21)$ with the patient in the left lateral position. We calculated the following parameters: left ventricular end-diastolic and end-systolic diameter indexed to body surface area, and left ventricular ejection fraction (LVEF; - biplane Simpson's rule). CO was computed from the classic formula: (left ventricular outflow tract area $\times$ velocity time integral) $\times \mathrm{HR} .{ }^{15)}$ For our purpose, $\mathrm{CO}$ was the average of 5 measurements. During the echo examination, BP was not measured. The same observer (RZ) performed all the echo examinations.

Biology: As usual in our facility, hemoglobin, B-type natriuretic peptide (BNP), uric acid, plasma sodium, plasma potassium, plasma nitrogen, and plasma creatinine were obtained. Glomerular filtration rate (GFR) was derived from the MDRD formula.

Statistical analyses: Data were checked for Gaussian distribution using the D'Agostino and Pearson Omnibus normality test. In appropriate instances, we tested differences using repeated measures ANOVA with the Tukey post-test if necessary. In some instances, we used the paired Student $t$ test. Correlation coefficients were derived from linear regression analysis. Agreement was assessed using the Bland-Altman method with the difference being plotted against the average and computation of the intra class correlation coefficient. In order to check for association between age, gender and presence of disease, a GLM ANOVA was performed. Data are expressed as the mean \pm standard deviation unless otherwise specified. A $P<0.05$ was considered significant. The statistical software packages used were GraphPad PRISM 4.0c for MacIntosh (May 2005) and GraphPad Software Inc. For the intra-class correlation coefficient and Kendall's W coefficient of concordance, we used tools from the Department of Obstetrics and Gynecology, Chinese University of Hong Kong (http://department.obg.cuhk. edu.hk/researchsupport). GLM ANOVA was performed using Statistica version 81984 - 2008 from StatSoft. 


\section{RESUlts}

Population: The study population consisted of 34 subjects. As shown in Table I, the subjects were rather young and the gender distribution was well balanced (47\% women). About half of the subjects studied had a normal heart. Most of the patients suffered from ischemic heart disease or systemic hypertension. At the time of the study, LV systolic function was moderately altered as suggested by the LVEF. Resting HR was normal and $\mathrm{BP}$ in the lower range of normality reflected at least some drug treatment effect. HR and LVEF contrasted somewhat with the functional class: patients were mostly in NYHA class II. Forty percent of the diseased subjects were treated with a betablocker and $42 \%$ with a renin angiotensin aldosterone system inhibitor. A statin was used in $34 \%$ of the diseased subjects. HR was similar between the 3 methods for the 20 patients who underwent the 3 different $\mathrm{CO}$ assessments: $P=0.69$; Repeated measures ANOVA. It was also similar when we analyzed the entire population who had had $\mathrm{CO}$ assessment using esCCO and IGR $(P=\mathrm{NS}$; paired Student $t$ test). No difference in BP pressure was found: $121 / 68 \mathrm{mmHg}$ for esCCO versus $123 / 72$ $\mathrm{mmHg}$ for IGR $(P=\mathrm{NS})$.

Laboratory data: Blood samples were drawn from the diseased subjects only. As reported in Table II, renal function was in or close to the normal range. BNP plasma concentration was also low, supporting the idea that the patients were controlled in terms of filling pressures.

Cardiac output measurements: $\mathrm{CO}$ obtained from VISMO ${ }^{\circledR}$ : esCCO was obtained in 27 subjects out of 34 , ie, $79.4 \%$ of the series. Those in whom esCCO was not available were patients with permanent atrial fibrillation or with a paced rhythm during the test. The esCCO and esCCI, averaged from 5 determinations, were $5.5 \pm 1.2 \mathrm{~L} /$ minute and $2.97 \pm 0.59 \mathrm{~L} / \mathrm{minute}^{2} \mathrm{~m}^{2}$, respectively. Both parameters were normally distributed.

The IGR method allowed also a successful measurement

Table I. Demographic Data

\begin{tabular}{lc}
\hline$n$ & 34 \\
Age (years) & $65 \pm 15$ \\
Female gender (\%) & $16(47)$ \\
Comorbidities & \\
\hline - HT (\%) & $23(70)$ \\
- Type 2 DM (\%) & $10(30)$ \\
- Tobacco (\%) & $12(33)$ \\
- Dyslipidemia (\%) & $19(60)$ \\
- Heredity (\%) & $1(3)$ \\
NYHA I/II/III (\%) & $10 / 64 / 26$ \\
Etiology of heart disease & \\
- Ischemic (\%) & $10(29)$ \\
- Post HT (\%) & $4(11)$ \\
- DCM (\%) & $3(9)$ \\
- CP (\%) & $1(3)$ \\
- Valvular heart disease & $1(3)$ \\
Normal heart (\%) & $15(44)$ \\
SBP (mmHg) & $103 \pm 17$ \\
DBP (mmHg) & $60 \pm 9$ \\
HR (bpm) & $60 \pm 15$ \\
LVEF $(\%)$ & $51 \pm 15$ \\
SpO (\%) & $97 \pm 1$ \\
\hline
\end{tabular}

HT indicates hypertension; NYHA, New York Heart Association; DCM, dilated cardiomyopathy; $\mathrm{CP}$, chronic pericarditis; SBP, systolic blood pressure; DBP, diastolic blood pressure; HR, heart rate; LVEF, left ventricular ejection fraction: and $\mathrm{SpO}_{2}, \mathrm{O}_{2}$ pulsatile saturation. in 27 subjects. Thus, the success rate was comparable with that of esCCO. The statistical distribution of IGR-derived $\mathrm{CO}$ was also normal. $\mathrm{CO}$ was $4.31 \pm 1.1 \mathrm{~L} /$ minute and the cardiac index was $2.39 \pm 0.67 \mathrm{~L} /$ minute $/ \mathrm{m}^{2}$. Most of the subjects had only one determination. In cases of an unsatisfactory test, a second determination was attempted, but this was uncommon. Testing was essentially not feasible in those patients unable to cope with the desirable respiratory rate.

Using 2D-Doppler echo, CO was satisfactorily obtained in 17 of the 20 diseased patients, ie, $85 \%$ of the studied sample. Once again, the statistical distribution was normal for both $\mathrm{CO}$ and $\mathrm{CI}$. Mean $\mathrm{CO}$ was $4.31 \pm 1.2 \mathrm{~L} /$ minute and $\mathrm{CI}$ was $2.85 \pm 0.72 \mathrm{~L} /$ minute $/ \mathrm{m}^{2}$. The 3 missing patients were those for whom the esCCO was not available, consequently, they did not undergo the echo examination. Thus, the 2D-Doppler echo is almost always feasible, unless the echogenicity of a patient is a limiting factor.

We were unable to show an effect of age, gender or presence of disease in our study population as suggested by GLM ANOVA $(P=$ NS $)$.

Inter-method correlation: The analysis showed that esCCO tended to overestimate CO compared to both 2D-Doppler echo and IGR $(P<0.0001$; repeated measures ANOVA with Tukey as post-test). IGR and 2D-Doppler echo data gave similar results $(P=\mathrm{NS})$. Despite this feature, linear regression between $\mathrm{CO}$ derived from esCCO and 2D-Doppler echo showed that they were highly correlated with an $r$ correlation coefficient of $0.79 ; P=0.0012$ (see Figure 1-A). CO computed by 2D-Doppler echo was better related to IGR-derived CO: $r$ correlation coefficient $=0.88 ; P<0.0001$ (Figure 1-B). However, we failed to find a significant relationship between esCCO and IGR-derived CO: $r=0.36 ; P=0.06$ - NS (Figure 1-C).

Inter-method agreement: Bland-Altman techniques allowed comparisons between pairs of methods: esCCO versus IGR, esCCO versus 2D-Doppler echo, and IGR versus 2D-Doppler echo. As shown in Figures 2-A and 2-B, there was a satisfactory agreement between esCCO and IGR for both $\mathrm{CO}$ and $\mathrm{CI}$. Bias was however rather large: $1.18 \pm 1.31 \mathrm{~L} /$ minute and the $95 \%$ limit of agreement was -0.39 to $3.76 \mathrm{~L} /$ minute for $\mathrm{CO}$. Considering CI, the bias was lower: $0.58 \pm 0.68 \mathrm{~L} / \mathrm{minute}^{2} \mathrm{~m}^{2}$ and the $95 \%$ limit of agreement was -0.74 to $1.9 \mathrm{~L} /$ minute $/ \mathrm{m}^{2}$. Though graphically the performances of the 2 methods were acceptable, the limit of agreement remained large for both parameters. As shown in Figures 2-C and 2-D, even though the same pattern was recorded between esCCO and 2D-Doppler echocardiography, the picture was more optimistic. For CO, the bias was once more quite large: $1.51 \pm 0.84 \mathrm{~L} /$ minute and the $95 \%$ limit of agreement -0.14 to $3.17 \mathrm{~L} /$ minute. For CI bias

Table II. Biological Data

\begin{tabular}{lc}
\hline$n$ & 20 \\
Pl Nitrogen (mmol/L) & $8.4 \pm 4.2$ \\
Pl Creatinine & $96 \pm 31$ \\
GFR (MDRD) & $72[52-86]$ \\
Hemoglobin $(\mathrm{g} / \mathrm{dL})$ & $14 \pm 1.29$ \\
HbAlc $(\%)$ & $6.1[5.65-6.65]$ \\
BNP $(\mathrm{ng} / \mathrm{L})$ & $121[42-215]$ \\
Pl Uric acid $(\mu \mathrm{mol} / \mathrm{L})$ & $480[380-513]$ \\
\hline
\end{tabular}

BNP indicates brain natriuretic peptide; $\mathrm{dL}$, deciliter; g, gram; GFR, glomerular filtration rate; HbAlc, glycated hemoglobin; L, liter; MDRD, Modification of Diet in Renal Disease; mmol, millimole; and Pl, plasma. 

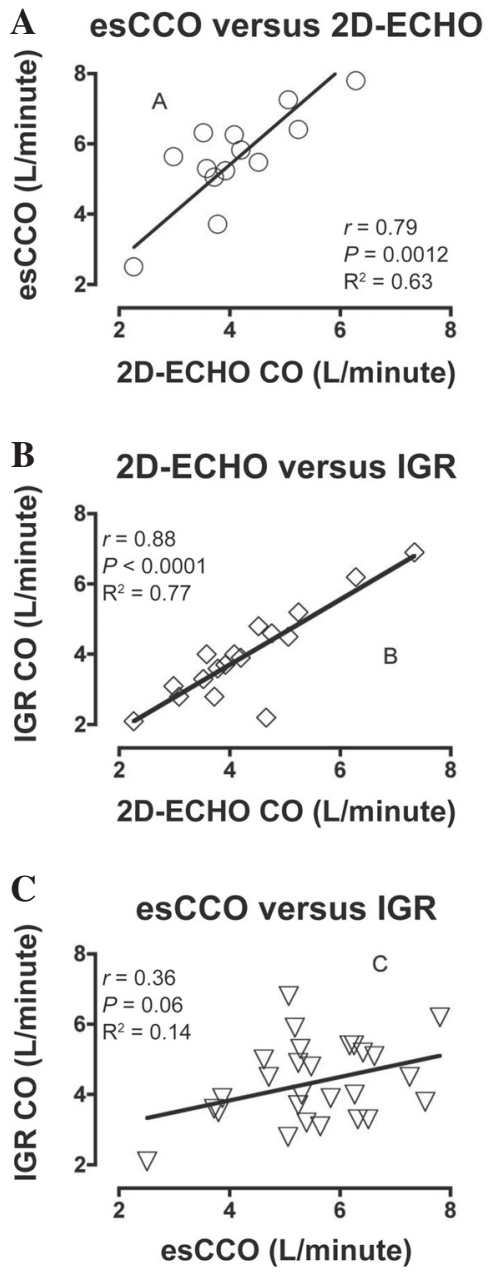

Figure 1. Analysis of correlation between estimating $\mathrm{CO}$ by esCCO versus 2D-Doppler echo (A), and versus IGR (C) and between 2D-Doppler echo estimated $\mathrm{CO}$ versus IGR (B).
A

CO: esCCO versus IGR

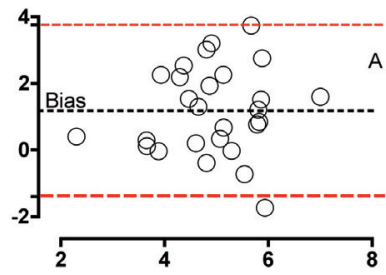

C

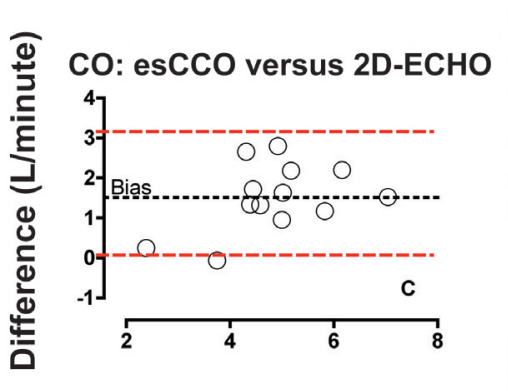

$\mathbf{E}$ CO: 2D-ECHO versus IGR

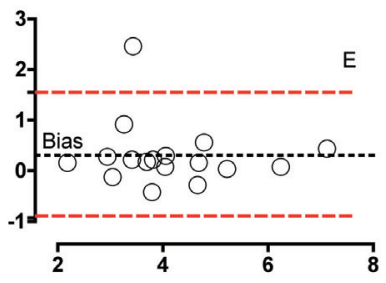

Average (L/minute)
B CI: esCCO versus IGR

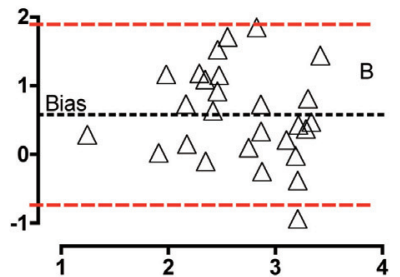

D

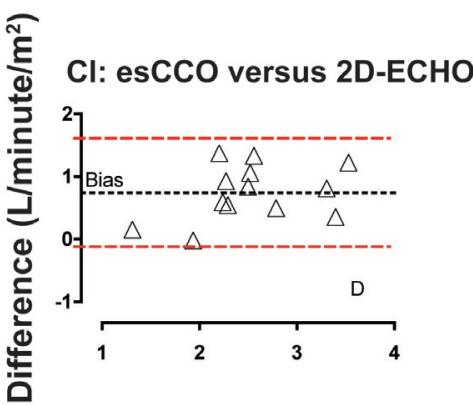

$\mathbf{F}$

CI: 2D-ECHO versus IGR

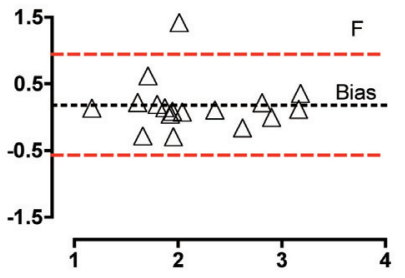

Average (L/minute $\left./ \mathrm{m}^{2}\right)$

Figure 2. Bland-Altman plots for CO and CI. A and B : esCCO versus IGR. C and D : esCCO versus 2D-Doppler echo. $\mathbf{E}$ and $\mathbf{F}$ : IGR versus 2D-Doppler echo.

was $0.74 \pm 0.44 \mathrm{~L} /$ minute $/ \mathrm{m}^{2}$ and the $95 \%$ limit of agreement was -0.12 to $1.61 \mathrm{~L} /$ minute $/ \mathrm{m}^{2}$. Figures $2-\mathrm{E}$ and $2-\mathrm{F}$ show $\mathrm{CO}$ and CI derived from 2D-Doppler echocardiography and IGR. The agreement was better in this case as depicted on the graphs: for $\mathrm{CO}$, the bias was $0.31 \pm 0.63 \mathrm{~L} /$ minute $(95 \%$ limit of agreement -0.93 to $1.55 \mathrm{~L} /$ minute) and for CI bias was 0.18 $\pm 0.39 \mathrm{~L} /$ minute $/ \mathrm{m}^{2}$ (95\% limit of agreement -0.58 to 0.94 ). According to our data, though the agreement seems satisfactory, esCCO tended to overestimate $\mathrm{CO}$ and $\mathrm{CI}$ whatever the comparative method. The small bias between 2D-Doppler echo and IGR argue for a better agreement between these 2 methods.

Intra-class correlation coefficient analysis allows the comparison of the 3 methods all together. We had only 13 patients remaining. In this case, the ICC was only 0.0042 , meaning that the inter-method agreement is at the very best modest, if any. Given the sample size when we considered the 3 methods together, we checked for an ICC between esCCO versus IGR, esCCO versus 2D-Doppler echo, and IGR versus 2DDoppler echo. The result remained unsatisfactory. However, when we checked for the ability of the 3 methods to classify correctly the patients in term of normal CI or not, the ICC was somewhat better (ICC $r$ coefficient $=0.56$ ) suggesting that the qualitative information provided is not related to the method used and therefore might be rather reliable. This was also underscored by the Kendall $\mathrm{W}$ coefficient of concordance between the 3 methods ( $\mathrm{W}=0.54 ; P=\mathrm{NS})$.

Reproducibility: We were able to analyze the reproducibility for esCCO and 2D-Doppler echocardiography. Reproducibility was correct with an ICC of 0.75 for esCCO and 0.68 for 2DDoppler echocardiography. The lesser performance of 2DDoppler echocardiography could be related to the intraobserver variability, which does not exist with an automatic method like VISMO $^{\circledR}$. The good concordance of repeated esCCO suggests that we could trust the data derived from this method for longitudinal assessment in such patients.

\section{Discussion}

The usefulness of calculating $\mathrm{CO}$ is established in the assessment of cardiovascular adaptation to exercise in physiolo- 
gy or in the top athlete, ${ }^{16)}$ as well as in various clinical situations, specifically in the perioperative setting. In patients with hemodynamic instability, $\mathrm{CO}$ is the most important element for the definition of shock. ${ }^{17)}$ The guidelines emphasize the value of close monitoring of hemodynamics after cardiac surgery. ${ }^{18)}$ In the case of patients with septic shock, CO monitoring should be considered to evaluate the introduction of positive inotropic drugs. ${ }^{19)}$ The estimation of hemodynamic parameters including cardiac index in subjects consulting for dyspnoea, helps to ascertain the diagnosis of heart failure with improved sensitivity and specificity compared to clinical and biological data. ${ }^{20)}$ Cardiac output allows also the calculation of cardiac power, which is a powerful prognostic factor in heart failure. ${ }^{21)}$ Accessing this parameter easily and reliably is therefore essential. Many methods are available nowadays such as bioimpedance. $^{16,20)}$

Pulse wave transit time derived esCCO method was compared with continuous thermodilution measurements in postoperative cardiac surgery patients. ${ }^{9)}$ The mean difference was only -0.06 and the precision was $0.82 \mathrm{~L} /$ minute. The accuracy of esCCO compared to thermodilution was recently reassessed in a multicenter setting and a large sample size. ${ }^{10)}$ Overall, the correlation coefficient between the 2 methods was $0.79(P<$ $0.0001)$. The bias and precision were $0.13 \mathrm{~L} /$ minute and 1.15 $\mathrm{L} /$ minute, respectively. The pulse contour analysis method seems to provide similar results whatever the technology used when compared to thermodilution. Thus the FloTrac system (Edwards Lifesciences) which requires a central or peripheral arterial catheter, showed a bias of $0.95 \mathrm{~L} /$ minute and precision of $1.41 \mathrm{~L} /$ minute. $^{22)}$ The PiCCO pulse contour system (Pulsion Medical Systems, Munich, Germany), which requires not only a central arterial catheter but also a subclavian or internal jugular catheter and a transpulmonary thermodilution calibration showed a bias of $-0.2 \mathrm{~L} / \mathrm{min}$ and precision of $1.2 \mathrm{~L} /$ minute. $^{23)}$ Another noninvasive arterial waveform analysis can be obtained using a Nexfin (BMEYE, Amsterdam, The Netherlands). ${ }^{24)}$ Compared to transthoracic Doppler echocardiography, the $r$ correlation coefficient was 0.68 and appeared to be in line with our result. However, the bias was lower, only 0.51 $\mathrm{L} /$ minute and the $95 \%$ limit of agreement ranged from -1.6 to $2.6 \mathrm{~L} /$ minute. Though the Nexfin differs from the VISMO ${ }^{\circledR}$, both methods are based on pulse contour analysis. Our results are close to those reported with Nexfin. In the intensive care setting, esCCO was compared to transthoracic echocardiography for CO measurements. ${ }^{25)}$ Though significant, the correlation coefficient between esCCO and transthoracic echocardiography (TTE) was lower than the one we reported: $0.61 ; P<$ 0.001 . Bland and Altman analysis showed a bias of $-1.6 \mathrm{~L} /$ minute and the limit of agreement ranged from -4.7 to $1.5 \mathrm{~L} /$ minute. The population studied was obviously different. However, the bias was very close to the one reported in our study and the limits of agreement similarly wide. Though there is agreement, it remains modest as stressed in a recent report ${ }^{26)}$ suggesting that these devices are not simply interchangeable when assessing $\mathrm{CO}$ in a research or clinical setting, an aspect also reflected by the ICC we report. In the same way, when we compared esCCO to IGR, we could not obtain better results. One could argue that the main reason could be the use of only one set of values for the IGR method. We must agree that the use of a single measurement with IGR remains a concern from a basic scientific standpoint. Peyton, et al ${ }^{27)}$ performed succes- sive paired measurements of pulmonary blood flow separated by a 5 minute interval using the IGR method in 8 patients preor postcardiac surgery, and in 8 healthy volunteers. They concluded that multiple measurements should be made and averaged when using the IGR technique for pulmonary blood flow determination. Thus, as suggested previously by Bland and Altman, ${ }^{28)}$ this aspect cannot be overlooked. We acknowledge that we had mostly only one measurement for costs reasons. The cost of a disposable product together with the expertise needed for the test execution and patient consent, specifically the elderly, are a concern. However, the bias between 2D-Doppler echo and the IGR method was only $0.31 \mathrm{~L} /$ minute in our series, a very satisfactory result similar to that reported elsewhere. ${ }^{29)}$ Neither a post-charge difference nor an obvious change in sympathetic tone (at least reflected by heart rate course) could explain our results. Though we admit a methodological limitation, it seems unlikely that having a single measurement could fully explain the weak agreement, if any, between esCCO and IGR in CO assessment. Others also reported an overestimation of $\mathrm{CO}$ by esCCO. ${ }^{10,25)}$ Various parameters such as differences in subject height, weight, heart rate, mean arterial pressure, systolic and diastolic blood pressure, and ventricular pre-ejection period (reflecting left ventricular isometric contraction time) have significant contributions to pulse wave transit time. ${ }^{30,31)}$ We did not observe any blood pressure or heart rate difference between the IGR and esCCO data. Blood pressure is a surrogate for systemic vascular resistance. The effect of systemic vascular resistance seems modest according to our data, which are in line with the report of Yamada, et al. ${ }^{10)}$ However, we did consider a single measure of heart rate and blood pressure not the trend in these parameters. Moreover, we did not analyse the contributions of the other variables that might explain our results. We also failed to show any effect of age, gender or presence of disease, however, the study population was small, with moderate alteration of LV systolic function and the patients were well controlled under treatment. Thus, these aspects limit any definite conclusion.

Though it seems difficult to switch from a method to another one, our study stressed that esCCO is a suitable alternative to assess $\mathrm{CO}$ and $\mathrm{CI}$ in stable patients, and equivalent to 2D-Doppler echocardiography and to a clear lesser degree to IGR. Moreover, the reproducibility of esCCO, which was slightly better than that of 2D-Doppler echocardiography-derived $\mathrm{CO}$, is at least acceptable. This suggests that both methods and especially esCCO might be suitable for screening and/ or monitoring purposes. This easy to use apparatus makes esC$\mathrm{CO}$ a valid method in stable patients or in the operating room. Notwithstanding that there is an overestimation of $\mathrm{CO}$ by esCCO compared with both IGR and 2D-Doppler echocardiography in our report, the ability of the 3 methods to correctly categorize subjects is quite satisfactory from a clinical standpoint. This aspect has not been clearly tested to our knowledge, but can be easily derived from the various papers devoted to the subject. ${ }^{17)}$

Conclusion: $\mathrm{CO}$ assessed by noninvasive methods is continuously developing. A number of noninvasive methods to determine the $\mathrm{CO}$ in a broad range of patients and settings are available. The esCCO derived from VISMO ${ }^{\circledR}$ is one of them. Though there is agreement with oldest ones, the esCCO seems to overestimate the $\mathrm{CO}$. Its internal reproducibility is satisfactory and it is able to correctly stratify the patients from their 
CO (ie, low or normal). Therefore, PWTT seems suitable for screening and/or monitoring purposes. Its accuracy needs to be reassessed in a larger population with various cardiac conditions. It would appear to be difficult to use this method in atrial fibrillation patients or in paced patients, subsets of patients which are of concern in daily practice.

Limitations of the study: The main limitation of our study is the sample size. Though this is explained by our study design (assessment of VISMO ${ }^{\circledR}$ versus IGR as a reference), the sample size must be increased. It would also be valuable to have multiple measurements of IGR-derived CO to screen the reproducibility of this method, but also to better assess the intermethod agreement. There is also a need to study a more heterogeneous population to ascertain the applicability of esCCO as provided by the VISMO ${ }^{\circledR}$ device.

\section{REFERENCES}

1. Mosterd A, Hoes AW, de Bruyne MC, et al. Prevalence of heart failure and left ventricular dysfunction in the general population; The Rotterdam Study. Eur Heart J 1999; 20: 447-55.

2. Laribi S, Aouba A, Nikolaou M, et al. Trends in death attributed to heart failure over the past two decades in Europe. Eur J Heart Fail 2012; 14: 234-9.

3. Swedberg K, Cleland J, Dargie H, et al. Guidelines for the diagnosis and treatment of chronic heart failure: executive summary (update 2005): Task Force for the Diagnosis and Treatment of Chronic Heart Failure of the European Society of Cardiology. Eur Heart J 2005; 26: 1115-40.

4. Binanay C, Califf RM, Hasselblad V, et al. Evaluation study of congestive heart failure and pulmonary artery catheterization effectiveness: the ESCAPE trial. JAMA 2005; 294: 1625-33.

5. Horster S, Stemmler HJ, Strecker N, et al. Cardiac Output Measurements in Septic Patients: Comparing the Accuracy of USCOM to PiCCO. Crit Care Res Pract 2012; 2012: 270631.

6. Koehler F, Winkler S, Schieber M, et al. Telemedical Interventional Monitoring in Heart Failure (TIM-HF), a randomized, controlled intervention trial investigating the impact of telemedicine on mortality in ambulatory patients with heart failure: study design. Eur J Heart Fail 2010; 12: 1354-62.

7. Lang CC, Karlin P, Haythe J, Tsao L, Mancini DM. Ease of noninvasive measurement of cardiac output coupled with peak VO2 determination at rest and during exercise in patients with heart failure. Am J Cardiol 2007; 99: 404-5.

8. Hofer CK, Ganter MT, Zollinger A. What technique should I use to measure cardiac output? Curr Opin Crit Care 2007; 13: 308-17. (Review)

9. Ishihara $\mathrm{H}$, Okawa $\mathrm{H}$, Tanabe $\mathrm{K}$, et al. A new non-invasive continuous cardiac output trend solely utilizing routine cardiovascular monitors. J Clin Monit Comput 2004; 18: 313-20.

10. Yamada T, Tsutsui M, Sugo Y, et al. Multicenter study verifying a method of noninvasive continuous cardiac output measurement using pulse wave transit time: a comparison with intermittent bolus termodilution cardiac output. Anesth Analg 2012; 115: 82-7.

11. Sugo Y, Ukawa T, Takeda S, Ishihara H, Kazama T, Takeda J. A novel continuous cardiac output monitor based on pulse wave transit time. Conf Proc IEEE Eng Med Biol Soc 2010; 2010 2853-6.

12. Gabrielsen A, Videbak R, Schou M, Damgaard M, Kastrup J, Norsk P. Non-invasive measurement of cardiac output in heart failure patients using a new foreign gas rebreathing technique. Clin Sci (Lond) 2002; 102: 247-52.

13. Stok WJ, Baisch F, Hillbrecht A, Schultz H, Meyer M, Karemaker JM. Noninvasive cardiac output measurement by arterial pulse analysis compared with inert gas rebreathing. J Appl Physiol 1993; 74: 2687-93
14. Villani F, Meazza R, Materazzo C. Non-invasive monitoring of cardiac hemodynamic parameters in doxorubicin-treated patients: comparison with echocardiography. Anticancer Res 2006; 26: 797-801.

15. Cheitlin MD, Armstrong WF, Aurigemma GP, et al. ACC/AHA/ ASE 2003 guideline update for the clinical application of echocardiography: summary article: a report of the American College of Cardiology/American Heart Association Task Force on Practice Guidelines (ACC/AHA/ASE Committee to Update the 1997 Guidelines for the Clinical Application of Echocardiography). Circulation 2003; 108: 1146-62.

16. Christensen TB, Jensen BV, Hjerpe J, Kanstrup IL. Cardiac output measured by electric bioimpedance compared with the $\mathrm{CO} 2$ rebreathing technique at different exercise levels. Clin Physiol 2000; 20: 101-5.

17. Marik PE, Baram M. Noninvasive hemodynamic monitoring in the intensive care unit. Crit Care Clin 2007; 23: 383-400. (Review)

18. Carl M, Alms A, Braun J, et al. Guidelines for intensive care in cardiac surgery patients: haemodynamic monitoring and cardiocirculatory treatment guidelines of the German Society for Thoracic and Cardiovascular Surgery and the German Society of Anaesthesiology and Intensive Care Medicine. Thorac Cardiovasc Surg 2007; 55: 130-48. (German)

19. Dellinger RP, Levy MM, Carlet JM, et al. Surviving Sepsis Campaign: International guidelines for management of severe sepsis and septic shock: 2008. Crit Care Med 2008; 36: 296-327.

20. Lo HY, Liao SC, Ng CJ, Kuan JT, Chen JC, Chiu TF. Utility of impedance cardiography for dyspneic patients in the ED. Am J Emerg Med 2007; 25: 437-41.

21. Cohen-Solal A, Tabet JY, Logeart D, Bourgoin P, Tokmakova M, Dahan M. A non-invasively determined surrogate of cardiac power ('circulatory power') at peak exercise is a powerful prognostic factor in chronic heart failure. Eur Heart J 2002; 10: 806-14.

22. Della Rocca G, Costa MG, Chiarandini P, et al. Arterial pulse cardiac output agreement with thermodilution in patients in hyperdynamic conditions. J Cardiothorac Vasc Anesth 2008; 22: 681-7.

23. Gödje $\mathrm{O}$, Höke K, Goetz AE, et al. Reliability of a new algorithm for continuous cardiac output determination by pulse-contour analysis during hemodynamic instability. Crit Care Med 2002; 30: $52-8$.

24. van der Spoel AG, Voogel AJ, Folkers A, Boer C, Bouwman RA. Comparison of noninvasive continuous arterial waveform analysis (Nexfin) with transthoracic Doppler echocardiography for monitoring of cardiac output. J Clin Anesth 2012; 24: 304-9.

25. Bataille B, Bertuit M, Mora M, et al. Comparison of esCCO and transthoracic echocardiography for non-invasive measurement of cardiac output intensive care. Br J Anaesth 2012; 109: 879-86.

26. Udy AA, Altukroni M, Jarrett P, Roberts JA, Lipman J. A comparison of pulse contour wave analysis and ultrasonic cardiac output monitoring in the critically ill. Anaesth Intensive Care 2012; 49: 631-7.

27. Peyton PJ, Chong SW. Minimally invasive measurement of cardiac output during surgery and critical care: a meta-analysis of accuracy and precision. Anesthesiology 2010; 113: 1220-35.

28. Bland JM, Altman DG. Measuring agreement in method comparison studies. Stat Methods Med Res 1999; 8: 135-60. (Review)

29. Sobański P, Sinkiewicz W, Kubica J, Blazejewski J, Bujak R. The reliability of noninvasive cardiac output measurement using the inert gas rebreathing method in patients with advanced heart failure. Cardiol J 2008; 15: 63-70.

30. Foo JY, Wilson SJ, Wang P. Factors that affect pulse wave time transmission in the monitoring of cardiovascular system. J Clin Monit Comput 2008; 22: 141-7.

31. Payne RA, Symeonides CN, Webb DJ, Maxwell SR. Pulse transit time measured from the ECG: an unreliable marker of beat-to-beat blood pressure. J Appl Physiol 2006; 100: 136-41. 